\title{
Existence of Solutions for Nonlinear Fractional Integro-Differential Equations with Three-Point Nonlocal Fractional Boundary Conditions
}

\author{
Ahmed Alsaedi and Bashir Ahmad \\ Department of Mathematics, Faculty of Science, King Abdulaziz University, P.O. Box 80203, \\ Jeddah 21589, Saudi Arabia \\ Correspondence should be addressed to Bashir Ahmad, bashir_qau@yahoo.com \\ Received 17 March 2010; Revised 6 May 2010; Accepted 11 June 2010 \\ Academic Editor: Kanishka Perera \\ Copyright (C 2010 A. Alsaedi and B. Ahmad. This is an open access article distributed under \\ the Creative Commons Attribution License, which permits unrestricted use, distribution, and \\ reproduction in any medium, provided the original work is properly cited. \\ We prove the existence and uniqueness of solutions for nonlinear integro-differential equations of \\ fractional order $q \in(1,2]$ with three-point nonlocal fractional boundary conditions by applying \\ some standard fixed point theorems.
}

\section{Introduction}

Fractional calculus (differentiation and integration of arbitrary order) is proved to be an important tool in the modelling of dynamical systems associated with phenomena such as fractal and chaos. In fact, this branch of calculus has found its applications in various disciplines of science and engineering such as mechanics, electricity, chemistry, biology, economics, control theory, signal and image processing, polymer rheology, regular variation in thermodynamics, biophysics, blood flow phenomena, aerodynamics, electro-dynamics of complex medium, viscoelasticity and damping, control theory, wave propagation, percolation, identification, and fitting of experimental data [1-4].

Recently, differential equations of fractional order have been addressed by several researchers with the sphere of study ranging from the theoretical aspects of existence and uniqueness of solutions to the analytic and numerical methods for finding solutions. For some recent work on fractional differential equations, see [5-11] and the references therein.

In this paper, we study the following nonlinear fractional integro-differential equations with three-point nonlocal fractional boundary conditions

$$
\begin{gathered}
D^{q} x(t)+f(t, x(t),(\phi x)(t),(\psi x)(t))=0, \quad 0<t<1,1<q \leq 2, \\
D^{(q-1) / 2} x(0)=0, \quad a D^{(q-1) / 2} x(1)+x(\eta)=0, \quad 0<\eta<1
\end{gathered}
$$


where $D$ is the standard Riemann-Liouville fractional derivative, $f:[0,1] \times X \times X \times X \rightarrow X$ is continuous, for $\gamma, \delta:[0,1] \times[0,1] \rightarrow[0, \infty)$,

$$
(\phi x)(t)=\int_{0}^{t} \gamma(t, s) x(s) d s, \quad(\psi x)(t)=\int_{0}^{t} \delta(t, s) x(s) d s,
$$

and $a \in \mathbb{R}$ satisfies the condition $a \Gamma(q)+\eta^{(q-1)} \Gamma((q+1) / 2) \neq 0$. Here, $(X,\|\cdot\|)$ is a Banach space and $C=C([0,1], X)$ denotes the Banach space of all continuous functions from $[0,1] \rightarrow X$ endowed with a topology of uniform convergence with the norm denoted by $\|\cdot\|$.

We remark that fractional boundary conditions result in the existence of both electric and magnetic surface currents on the strip and are similar to the impedance boundary conditions with pure imaginary impedance, and in the physical optics approximation, the ratio of the surface currents is the same as for the impedance strip. For the comparison of the physical characteristics of the fractional and impedance strips such as radiation pattern, monostatic radar cross-section, and surface current densities, see [12]. The concept of nonlocal multipoint boundary conditions is quite important in various physical problems of applied nature when the controllers at the end points of the interval (under consideration) dissipate or add energy according to the censors located at intermediate points. Some recent results on nonlocal fractional boundary value problems can be found in [13-15].

\section{Preliminaries}

Let us recall some basic definitions [1-3] on fractional calculus.

Definition 2.1. The Riemann-Liouville fractional integral of order $q$ is defined as

$$
I^{q} g(t)=\frac{1}{\Gamma(q)} \int_{0}^{t} \frac{g(s)}{(t-s)^{1-q}} d s, \quad q>0
$$

provided the integral exists.

Definition 2.2. The Riemann-Liouville fractional derivative of order $q$ for a function $g(t)$ is defined by

$$
D^{q} g(t)=\frac{1}{\Gamma(n-q)}\left(\frac{d}{d t}\right)^{n} \int_{0}^{t} \frac{g(s)}{(t-s)^{q-n+1}} d s, \quad n-1<q \leq n, q>0
$$

provided the right-hand side is pointwise defined on $(0, \infty)$.

Lemma 2.3 (see [16]). For $q>0$, let $x, D^{q} x \in C(0,1) \cap L(0,1)$. Then

$$
I^{q} D^{q} x(t)=x(t)+c_{1} t^{q-1}+c_{2} t^{q-2}+\cdots+c_{n} t^{q-n},
$$

where $c_{i} \in \mathbb{R}, i=1,2, \ldots, n$ ( $n$ is the smallest integer such that $n \geq q$ ). 
Lemma 2.4 (see [2]). Let $x \in L(0,1)$. Then

(i) $D^{v} I^{\mu} x(t)=I^{\mu-v} x(t), \mu>v>0$;

(ii) $D^{\mu} t^{\xi-1}=(\Gamma(\xi) / \Gamma(\xi-\mu)) t^{\xi-\mu-1}, \mu>0, \xi>0$.

Lemma 2.5. For a given $\sigma \in C[0,1] \cap L(0,1)$, the unique solution of the boundary value problem

$$
\begin{gathered}
D^{q} x(t)+\sigma(t)=0, \quad 0<t<1,1<q \leq 2, \\
D^{(q-1) / 2} x(0)=0, \quad a D^{(q-1) / 2} x(1)+x(\eta)=0,0<\eta<1,
\end{gathered}
$$

is given by

$$
\begin{aligned}
x(t)=- & \int_{0}^{t} \frac{(t-s)^{q-1}}{\Gamma(q)} \sigma(s) d s+\frac{\Gamma((q+1) / 2) t^{q-1}}{\left[a \Gamma(q)+\eta^{(q-1)} \Gamma((q+1) / 2)\right]} \\
& \times\left\{\int_{0}^{\eta} \frac{(\eta-s)^{q-1}}{\Gamma(q)} \sigma(s) d s+a \int_{0}^{1} \frac{(1-s)^{(q-1) / 2}}{\Gamma((q+1) / 2)} \sigma(s) d s\right\} .
\end{aligned}
$$

Proof. In view of Lemma 2.3, the fractional differential equation in (2.4) is equivalent to the integral equation

$$
x(t)=-I^{q} \sigma(t)+b_{1} t^{q-1}+b_{2} t^{q-2}=-\int_{0}^{t} \frac{(t-s)^{q-1}}{\Gamma(q)} \sigma(s) d s+b_{1} t^{q-1}+b_{2} t^{q-2},
$$

where $b_{1}, b_{2} \in \mathbb{R}$ are arbitrary constants. Applying the boundary conditions for (2.4), we find that $b_{2}=0$ and

$$
b_{1}=\frac{\Gamma((q+1) / 2)}{\left[a \Gamma(q)+\eta^{(q-1)} \Gamma((q+1) / 2)\right]}\left(\int_{0}^{\eta} \frac{(\eta-s)^{q-1}}{\Gamma(q)} \sigma(s) d s+a \int_{0}^{1} \frac{(1-s)^{(q-1) / 2}}{\Gamma((q+1) / 2)} \sigma(s) d s\right) .
$$

Substituting the values of $b_{1}$ and $b_{2}$ in (2.6), we obtain (2.5). This completes the proof.

\section{Main Results}

To establish the main results, we need the following assumptions.

$\left(\mathrm{A}_{1}\right)$ There exist positive functions $L_{1}(t), L_{2}(t), L_{3}(t)$ such that

$$
\begin{aligned}
& \|f(t, x(t),(\phi x)(t),(\psi x)(t))-f(t, y(t),(\phi y)(t),(\psi y)(t))\| \\
& \quad \leq L_{1}(t)\|x-y\|+L_{2}(t)\|\phi x-\phi y\|+L_{3}(t)\|\psi x-\psi y\|, \quad \forall t \in[0,1], x, y \in X .
\end{aligned}
$$


Further,

$$
\begin{gathered}
r_{0}=\sup _{t \in[0,1]}\left|\int_{0}^{t} \gamma(t, s) d s\right|, \quad \delta_{0}=\sup _{t \in[0,1]}\left|\int_{0}^{t} \delta(t, s) d s\right|, \\
I_{L}^{q}=\sup _{t \in[0,1]}\left\{\left|I^{q} L_{1}(t)\right|,\left|I^{q} L_{2}(t)\right|,\left|I^{q} L_{3}(t)\right|\right\}, \\
I^{(q+1) / 2} L(1)=\max \left\{\left|I^{(q+1) / 2} L_{1}(1)\right|,\left|I^{(q+1) / 2} L_{2}(1)\right|,\left|I^{(q+1) / 2} L_{3}(1)\right|\right\}, \\
I^{q} L(\eta)=\max \left\{\left|I^{q} L_{1}(\eta)\right|,\left|I^{q} L_{2}(\eta)\right|,\left|I^{q} L_{3}(\eta)\right|\right\} .
\end{gathered}
$$

$\left(\mathrm{A}_{2}\right)$ There exists a number $\kappa$ such that $\Lambda \leq \kappa<1$, where

$$
\begin{aligned}
& \Lambda=\left(1+\gamma_{0}+\delta_{0}\right)\left\{I_{L}^{q}+\lambda_{1}\left(I^{q} L(\eta)+|a| I^{(q+1) / 2} L(1)\right)\right\} \\
& \lambda_{1}=\frac{\Gamma((q+1) / 2)}{\left[a \Gamma(q)+\eta^{(q-1)} \Gamma((q+1) / 2)\right]} .
\end{aligned}
$$

$\left(\mathrm{A}_{3}\right)\|f(t, x(t),(\phi x)(t),(\psi x)(t))\| \leq \mu(t)$, for all $(t, x, \phi x, \psi x) \in[0,1] \times X \times X \times X, \mu \in$ $L^{1}\left([0,1], R^{+}\right)$.

Theorem 3.1. Assume that $f:[0,1] \times X \times X \times X \rightarrow X$ is a jointly continuous function and satisfies the assumption $\left(A_{1}\right)$. Then the boundary value problem (1.1) has a unique solution provided $\Lambda<1$, where $\Lambda$ is given in the assumption $\left(A_{2}\right)$.

Proof. Define $\digamma: C \rightarrow C$ by

$$
\begin{aligned}
(\digamma x)(t)=- & \int_{0}^{t} \frac{(t-s)^{q-1}}{\Gamma(q)} f(s, x(s),(\phi x)(s),(\psi x)(s)) d s \\
& +\frac{\Gamma((q+1) / 2) t^{q-1}}{\left[a \Gamma(q)+\eta^{(q-1)} \Gamma((q+1) / 2)\right]} \\
& \times\left\{\int_{0}^{\eta} \frac{(\eta-s)^{q-1}}{\Gamma(q)} f(s, x(s),(\phi x)(s),(\psi x)(s)) d s\right. \\
& \left.+a \int_{0}^{1} \frac{(1-s)^{(q-1) / 2}}{\Gamma((q+1) / 2)} f(s, x(s),(\phi x)(s),(\psi x)(s)) d s\right\}, \quad t \in[0,1] .
\end{aligned}
$$

Let us set $\sup _{t \in[0,1]}|f(t, 0,0,0)|=M$, and choose

$$
r \geq \frac{M}{(1-\lambda)}\left\{\frac{1+\lambda_{1} \eta^{q}}{\Gamma(q+1)}+\frac{\lambda_{1}|a|}{\Gamma((q+3) / 2)}\right\}
$$


where $\lambda$ is such that $\Lambda \leq \lambda<1$. Now we show that $\digamma B_{r} \subset B_{r}$, where $B_{r}=\{x \in C:\|x\| \leq r\}$. For $x \in B_{r}$, we have

$$
\begin{aligned}
& \|(\digamma x)(t)\| \\
& \leq \int_{0}^{t} \frac{(t-s)^{q-1}}{\Gamma(q)}\|f(s, x(s),(\phi x)(s),(\psi x)(s))\| d s \\
& +\left|\frac{\Gamma((q+1) / 2) t^{q-1}}{\left[a \Gamma(q)+\eta^{(q-1)} \Gamma((q+1) / 2)\right]}\right| \\
& \times\left\{\int_{0}^{\eta} \frac{(\eta-s)^{q-1}}{\Gamma(q)}\|f(s, x(s),(\phi x)(s),(\psi x)(s))\| d s\right. \\
& \left.+|a| \int_{0}^{1} \frac{(1-s)^{(q-1) / 2}}{\Gamma((q+1) / 2)}\|f(s, x(s),(\phi x)(s),(\psi x)(s))\| d s\right\} \\
& \leq \int_{0}^{t} \frac{(t-s)^{q-1}}{\Gamma(q)}(\|f(s, x(s),(\phi x)(s),(\psi x)(s))-f(s, 0,0,0)\|+\|f(s, 0,0,0)\|) d s \\
& +\left|\frac{\Gamma((q+1) / 2) t^{q-1}}{\left[a \Gamma(q)+\eta^{(q-1)} \Gamma((q+1) / 2)\right]}\right| \\
& \times\left\{\int_{0}^{\eta} \frac{(\eta-s)^{q-1}}{\Gamma(q)}(\|f(s, x(s),(\phi x)(s),(\psi x)(s))-f(s, 0,0,0)\|+\|f(s, 0,0,0)\|) d s\right. \\
& \left.+|a| \int_{0}^{1} \frac{(1-s)^{(q-1) / 2}}{\Gamma((q+1) / 2)}(\|f(s, x(s),(\phi x)(s),(\psi x)(s))-f(s, 0,0,0)\|+\|f(s, 0,0,0)\|) d s\right\} \\
& \leq \int_{0}^{t} \frac{(t-s)^{q-1}}{\Gamma(q)}\left(L_{1}(s)\|x(s)\|+L_{2}(s)\|(\phi x)(s)\|+L_{3}(s)\|(\psi x)(s)\|+M\right) d s \\
& +\left|\frac{\Gamma((q+1) / 2) t^{q-1}}{\left[a \Gamma(q)+\eta^{(q-1)} \Gamma((q+1) / 2)\right]}\right| \\
& \times\left\{\int_{0}^{\eta} \frac{(\eta-s)^{q-1}}{\Gamma(q)}\left(L_{1}(s)\|x(s)\|+L_{2}(s)\|(\phi x)(s)\|+L_{3}(s)\|(\psi x)(s)\|+M\right) d s\right. \\
& \left.+|a| \int_{0}^{1} \frac{(1-s)^{(q-1) / 2}}{\Gamma((q+1) / 2)}\left(L_{1}(s)\|x(s)\|+L_{2}(s)\|(\phi x)(s)\|+L_{3}(s)\|(\psi x)(s)\|+M\right) d s\right\} \\
& \leq \int_{0}^{t} \frac{(t-s)^{q-1}}{\Gamma(q)}\left(L_{1}(s)\|x(s)\|+\gamma_{0} L_{2}(s)\|x(s)\|+\delta_{0} L_{3}(s)\|x(s)\|+M\right) d s \\
& +\left|\frac{\Gamma((q+1) / 2) t^{q-1}}{\left[a \Gamma(q)+\eta^{(q-1)} \Gamma((q+1) / 2)\right]}\right|
\end{aligned}
$$




$$
\begin{aligned}
\times & \left\{\int_{0}^{\eta} \frac{(\eta-s)^{q-1}}{\Gamma(q)}\left(L_{1}(s)\|x(s)\|+\gamma_{0} L_{2}(s)\|x(s)\|+\delta_{0} L_{3}(s)\|x(s)\|+M\right) d s\right. \\
& \left.+|a| \int_{0}^{1} \frac{(1-s)^{(q-1) / 2}}{\Gamma((q+1) / 2)}\left(L_{1}(s)\|x(s)\|+\gamma_{0} L_{2}(s)\|x(s)\|+\delta_{0} L_{3}(s)\|x(s)\|+M\right) d s\right\} \\
\leq & \left(I^{q} L_{1}(t)+\gamma_{0} I^{q} L_{2}(t)+\delta_{0} I^{q} L_{3}(t)\right) r+\frac{M t^{q}}{\Gamma(q+1)}+\left|\frac{\Gamma((q+1) / 2) t^{q-1}}{\left[a \Gamma(q)+\eta^{(q-1)} \Gamma((q+1) / 2)\right]}\right| \\
\times & \left\{\left(\left(I^{q} L_{1}(\eta)+\gamma_{0} I^{q} L_{2}(\eta)+\delta_{0} I^{q} L_{3}(\eta)\right) r+\frac{M \eta^{q}}{\Gamma(q+1)}\right)\right. \\
& \left.+|a|\left(\left(I^{(q+1) / 2} L_{1}(1)+\gamma_{0} I^{(q+1) / 2} L_{2}(1)+\delta_{0} I^{(q+1) / 2} L_{3}(1)\right) r+\frac{M}{\Gamma((q+3) / 2)}\right)\right\} \\
\leq & \left(1+\gamma_{0}+\delta_{0}\right)\left\{I_{L}^{q}+\lambda_{1}\left(I^{q} L(\eta)+|a| I^{(q+1) / 2} L(1)\right)\right\} r+M\left\{\frac{1+\lambda_{1} \eta^{q}}{\Gamma(q+1)}+\frac{\lambda_{1}|a|}{\Gamma((q+3) / 2)}\right\} \\
\leq & (\Lambda+1-\lambda) r \leq r .
\end{aligned}
$$

Now, for $x, y \in C$ and for each $t \in[0,1]$, we obtain

$$
\begin{aligned}
& \|(\digamma x)(t)-(\digamma y)(t)\| \\
& \leq \int_{0}^{t} \frac{(t-s)^{q-1}}{\Gamma(q)}\|f(s, x(s),(\phi x)(s),(\psi x)(s))-f(s, y(s),(\phi y)(s),(\psi y)(s))\| d s \\
& +\left|\frac{\Gamma((q+1) / 2) t^{q-1}}{\left[a \Gamma(q)+\eta^{(q-1)} \Gamma((q+1) / 2)\right]}\right| \\
& \quad \times\left\{\int_{0}^{\eta} \frac{(\eta-s)^{q-1}}{\Gamma(q)}\|f(s, x(s),(\phi x)(s),(\psi x)(s))-f(s, y(s),(\phi y)(s),(\psi y)(s))\| d s\right. \\
& \left.\quad+|a| \int_{0}^{1} \frac{(1-s)^{(q-1) / 2}}{\Gamma((q+1) / 2)}\|f(s, x(s),(\phi x)(s),(\psi x)(s))-f(s, y(s),(\phi y)(s),(\psi y)(s))\| d s\right\} \\
& \leq \int_{0}^{t} \frac{(t-s)^{q-1}}{\Gamma(q)}\left(L_{1}(s)\|x-y\|+L_{2}(s)\|\phi x-\phi y\|+L_{3}(s)\|\psi x-\psi y\|\right) d s \\
& \quad+\left|\frac{\Gamma((q+1) / 2) t^{q-1}}{\left[a \Gamma(q)+\eta^{(q-1)} \Gamma((q+1) / 2)\right]}\right| \\
& \quad \times\left\{\int_{0}^{\eta} \frac{(\eta-s)^{q-1}}{\Gamma(q)}\left(L_{1}(s)\|x-y\|+L_{2}(s)\|\phi x-\phi y\|+L_{3}(s)\|\psi x-\psi y\|\right) d s\right. \\
& \left.\quad+|a| \int_{0}^{1} \frac{(1-s)^{(q-1) / 2}}{\Gamma((q+1) / 2)}\left(L_{1}(s)\|x-y\|+L_{2}(s)\|\phi x-\phi y\|+L_{3}(s)\|\psi x-\psi y\|\right) d s\right\}
\end{aligned}
$$




$$
\begin{aligned}
\leq & \left(I^{q} L_{1}(t)+\gamma_{0} I^{q} L_{2}(t)+\delta_{0} I^{q} L_{3}(t)\right)\|x-y\|+\left|\frac{\Gamma((q+1) / 2) t^{q-1}}{\left[a \Gamma(q)+\eta^{(q-1)} \Gamma((q+1) / 2)\right]}\right| \\
& \times\left\{I^{q} L_{1}(\eta)+\gamma_{0} I^{q} L_{2}(\eta)+\delta_{0} I^{q} L_{3}(\eta)\right. \\
& \left.\quad+|a|\left(I^{(q+1) / 2} L_{1}(1)+\gamma_{0} I^{(q+1) / 2} L_{2}(1)+\delta_{0} I^{(q+1) / 2} L_{3}(1)\right)\right\}\|x-y\| \\
\leq & \left(1+\gamma_{0}+\delta_{0}\right)\left\{I_{L}^{q}+\lambda_{1}\left(I^{q} L(\eta)+|a| I^{(q+1) / 2} L(1)\right)\right\}\|x-y\| \\
= & \Lambda\|x-y\|,
\end{aligned}
$$

where we have used the assumption $\left(\mathrm{A}_{2}\right)$. As $\Lambda<1$, therefore $\digamma$ is a contraction. Thus, the conclusion of the theorem follows by the contraction mapping principle.

Now, we state Krasnoselskii's fixed point theorem [17] which is needed to prove the following result to prove the existence of at least one solution of (1.1).

Theorem 3.2. Let $M$ be a closed convex and nonempty subset of a Banach space $X$. Let $A, B$ be the operators such that (i) $A x+B y \in M$ whenever $x, y \in M$; (ii) $A$ is compact and continuous; (iii) $B$ is a contraction mapping. Then there exists $z \in M$ such that $z=A z+B z$.

Theorem 3.3. Let $f:[0,1] \times X \times X \times X \rightarrow X$ be jointly continuous, and the assumptions $\left(A_{1}\right)$ and $\left(A_{3}\right)$ hold with

$$
\Lambda_{1}=\lambda_{1}\left(1+\gamma_{0}+\delta_{0}\right)\left(I^{q} L(\eta)+|a| I^{(q+1) / 2} L(1)\right)<1
$$

Then there exists at least one solution of the boundary value problem $(1.1)$ on $[0,1]$.

Proof. Let us fix

$$
r \geq\|\mu\|_{L^{1}}\left\{\frac{1+\lambda_{1} \eta^{q}}{\Gamma(q+1)}+\frac{\lambda_{1}|a|}{\Gamma((q+3) / 2)}\right\}
$$

and consider $B_{r}=\{x \in C:\|x\| \leq r\}$. We define the operators $\Theta_{1}$ and $\Theta_{2}$ on $B_{r}$ as

$$
\begin{aligned}
\left(\Theta_{1} x\right)(t)= & -\int_{0}^{t} \frac{(t-s)^{q-1}}{\Gamma(q)} f(s, x(s),(\phi x)(s),(\psi x)(s)) d s \\
\left(\Theta_{2} x\right)(t)= & \frac{\Gamma((q+1) / 2) t^{q-1}}{\left[a \Gamma(q)+\eta^{(q-1)} \Gamma((q+1) / 2)\right]} \\
& \times\left\{\int_{0}^{\eta} \frac{(\eta-s)^{q-1}}{\Gamma(q)} f(s, x(s),(\phi x)(s),(\psi x)(s)) d s\right. \\
& \left.+a \int_{0}^{1} \frac{(1-s)^{(q-1) / 2}}{\Gamma((q+1) / 2)} f(s, x(s),(\phi x)(s),(\psi x)(s)) d s\right\} .
\end{aligned}
$$


For $x, y \in B_{r}$, we find that

$$
\left\|\Theta_{1} x+\Theta_{2} y\right\| \leq\|\mu\|_{L^{1}}\left\{\frac{1+\lambda_{1} \eta^{q}}{\Gamma(q+1)}+\frac{\lambda_{1}|a|}{\Gamma((q+3) / 2)}\right\} \leq r .
$$

Thus, $\Theta_{1} x+\Theta_{2} y \in B_{r}$. It follows from the assumption $\left(A_{1}\right)$ that $\Theta_{2}$ is a contraction mapping for $\Lambda_{1}<1$.

In order to prove that $\Theta_{1}$ is compact and continuous, we follow the approach used in [6,7]. Continuity of $f$ implies that the operator $\left(\Theta_{1} x\right)(t)$ is continuous. Also, $\left(\Theta_{1} x\right)(t)$ is uniformly bounded on $B_{r}$ as

$$
\left\|\Theta_{1} x\right\| \leq \frac{\|\mu\|_{L^{1}}}{\Gamma(q+1)} .
$$

Now, we show that $\left(\Theta_{1} x\right)(t)$ is equicontinuous. Since $f$ is bounded on the compact set $[0,1] \times B_{r} \times B_{r} \times B_{r}$, therefore, we define $\sup _{(t, x, \phi x, \psi x) \in[0,1] \times B_{r} \times B_{r} \times B_{r}}\|f(t, x, \phi x, \psi x)\|=f_{\text {max }}$. Consequently, for $t_{1}, t_{2} \in[0,1]$, we have

$$
\begin{aligned}
& \left\|\left(\Theta_{1} x\right)\left(t_{1}\right)-\left(\Theta_{1} x\right)\left(t_{2}\right)\right\| \\
& =\| \frac{1}{\Gamma(q)} \int_{0}^{t_{1}}\left(\left(t_{2}-s\right)^{q-1}-\left(t_{1}-s\right)^{q-1}\right) f(s, x(s), \phi x(s), \psi x(s)) d s \\
& \quad+\int_{t_{1}}^{t_{2}}\left(t_{2}-s\right)^{q-1} f(s, x(s), \phi x(s), \psi x(s)) d s \| \\
& \leq \frac{f_{\max }}{\Gamma(q+1)}\left|2\left(t_{2}-t_{1}\right)^{q}+t_{1}^{q}-t_{2}^{q}\right|
\end{aligned}
$$

which is independent of $x$. So, $\Theta_{1}$ is relatively compact on $B_{r}$. Hence, By Arzela-Ascoli's Theorem, $\Theta_{1}$ is compact on $B_{r}$. Thus all the assumptions of Theorem 3.2 are satisfied and the conclusion of Theorem 3.2 implies that the boundary value problem (1.1) has at least one solution on $[0,1]$.

Example. Consider the following boundary value problem:

$$
\begin{gathered}
{ }^{c} D^{3 / 2} x(t)+\frac{t}{8} \frac{|x|}{1+|x|}+\frac{1}{5} \int_{0}^{t} \frac{e^{-(s-t)}}{5} x(s) d s+\frac{1}{5} \int_{0}^{t} \frac{e^{-(s-t) / 2}}{5} x(s) d s=0, \quad t \in[0,1] \\
D^{1 / 4} x(0)=0, \quad a D^{1 / 4} x(1)+x\left(\frac{1}{3}\right)=0 .
\end{gathered}
$$


Here, $q=3 / 2, \gamma(t, s)=e^{-(s-t)} / 5, \delta=e^{-(s-t) / 2} / 5, a=1, \eta=1 / 3$. With $\gamma_{0}=(e-1) / 5, \delta_{0}=$ $2(\sqrt{e}-1) / 5$, we find that

$$
\Lambda=\frac{8(e+2(\sqrt{e}+1))(9 \sqrt{3 \pi}+\Gamma(1 / 4))}{225 \sqrt{\pi}(2 \sqrt{3 \pi}+\Gamma(1 / 4))}<1 .
$$

Thus, by Theorem 3.1, the boundary value problem (3.14) has a unique solution on $[0,1]$.

\section{Conclusions}

This paper studies the existence and uniqueness of solutions for nonlinear integro-differential equations of fractional order $q \in(1,2]$ with three-point nonlocal fractional boundary conditions involving the fractional derivative $D^{(q-1) / 2} x(\cdot)$. Our results are based on a generalized variant of Lipschitz condition given in $\left(\mathrm{A}_{1}\right)$, that is, there exist positive functions $L_{1}(t), L_{2}(t)$, and $L_{3}(t)$ such that

$$
\begin{aligned}
& \|f(t, x(t),(\phi x)(t),(\psi x)(t))-f(t, y(t),(\phi y)(t),(\psi y)(t))\| \\
& \quad \leq L_{1}(t)\|x-y\|+L_{2}(t)\|\phi x-\phi y\|+L_{3}(t)\|\psi x-\psi y\|, \quad \forall t \in[0,1], x, y \in X .
\end{aligned}
$$

In case $L_{1}(t), L_{2}(t)$, and $L_{3}(t)$ are constant functions, that is, $L_{1}(t)=L_{1}, L_{2}(t)=L_{2}$, and $L_{3}(t)=$ $L_{3}\left(L_{1}, L_{2}\right.$, and $L_{3}$ are positive real numbers), then Lipschitz-generalized variant reduces to the classical Lipschitz condition and $\Lambda$ in the assumption $\left(\mathrm{A}_{2}\right)$ takes the form

$$
\Lambda=\left(L_{1}+\gamma_{0} L_{2}+\delta_{0} L_{3}\right)\left\{\frac{1+\lambda_{1} \eta^{q}}{\Gamma(q+1)}+\frac{\lambda_{1}|a|}{\Gamma((q+3) / 2)}\right\} .
$$

In the limit $q \rightarrow 2$, our results correspond to a second-order integro-differential equation with fractional boundary conditions:

$$
\begin{gathered}
D^{2} x(t)+f(t, x(t),(\phi x)(t),(\psi x)(t))=0, \quad 0<t<1, \\
D^{1 / 2} x(0)=0, \quad a D^{1 / 2} x(1)+x(\eta)=0, \quad 0<\eta<1 .
\end{gathered}
$$

\section{Acknowledgment}

The authors are grateful to the referees for their careful review of the manuscript.

\section{References}

[1] S. G. Samko, A. A. Kilbas, and O. I. Marichev, Fractional Integrals and Derivatives, Gordon and Breach Science, Yverdon, Switzerland, 1993.

[2] I. Podlubny, Fractional Differential Equations, vol. 198 of Mathematics in Science and Engineering, Academic Press, San Diego, Calif, USA, 1999. 
[3] A. A. Kilbas, H. M. Srivastava, and J. J. Trujillo, Theory and Applications of Fractional Differential Equations, vol. 204 of North-Holland Mathematics Studies, Elsevier Science B.V., Amsterdam, The Netherlands, 2006.

[4] J. Sabatier, O. P. Agrawal, and J. A. T. Machad, Eds., Advances in Fractional Calculus: Theoretical Developments and Applications in Physics and Engineering, Springer, Dordrecht, The Netherlands, 2007.

[5] V. Daftardar-Gejji and S. Bhalekar, "Boundary value problems for multi-term fractional differential equations," Journal of Mathematical Analysis and Applications, vol. 345, no. 2, pp. 754-765, 2008.

[6] G. M. N'Guérékata, "A Cauchy problem for some fractional abstract differential equation with non local conditions," Nonlinear Analysis: Theory, Methods \& Applications, vol. 70, no. 5, pp. 1873-1876, 2009.

[7] G. M. Mophou and G. M. N'Guérékata, "On integral solutions of some nonlocal fractional differential equations with nondense domain," Nonlinear Analysis: Theory, Methods $\mathcal{E}$ Applications, vol. 71, no. 10, pp. 4668-4675, 2009.

[8] V. Lakshmikantham, S. Leela, and J. V. Devi, Theory of Fractional Dynamic Systems, Cambridge Academic Publishers, Cambridge, UK, 2009.

[9] V. Gafiychuk and B. Datsko, "Mathematical modeling of different types of instabilities in time fractional reaction-diffusion systems," Computers $\mathcal{E}$ Mathematics with Applications, vol. 59, no. 3, pp. 1101-1107, 2010.

[10] G. M. Mophou, "Existence and uniqueness of mild solutions to impulsive fractional differential equations," Nonlinear Analysis: Theory, Methods E Applications, vol. 72, no. 3-4, pp. 1604-1615, 2010.

[11] B. Ahmad, "Existence of solutions for irregular boundary value problems of nonlinear fractional differential equations," Applied Mathematics Letters, vol. 23, no. 4, pp. 390-394, 2010.

[12] E. I. Veliev, M. V. Ivakhnychenko, and T. M. Ahmedov, "scattering properties of the strip with fractional boundary conditions and comparison with the impedance strip," Progress In Electromagnetics Research C, vol. 2, pp. 189-205, 2008.

[13] B. Ahmad and J. J. Nieto, "Existence of solutions for nonlocal boundary value problems of higherorder nonlinear fractional differential equations," Abstract and Applied Analysis, vol. 2009, Article ID 494720, 9 pages, 2009.

[14] B. Ahmad and J. R. Graef, "Coupled systems of nonlinear fractional differential equations with nonlocal boundary conditions," PanAmerican Mathematical Journal, vol. 19, no. 3, pp. 29-39, 2009.

[15] B. Ahmad and J. J. Nieto, "Existence results for a coupled system of nonlinear fractional differential equations with three-point boundary conditions," Computers \& Mathematics with Applicationsl, vol. 58, no. 9, pp. 1838-1843, 2009.

[16] Z. Bai and H. Lü, "Positive solutions for boundary value problem of nonlinear fractional differential equation," Journal of Mathematical Analysis and Applications, vol. 311, no. 2, pp. 495-505, 2005.

[17] D. R. Smart, Fixed Point Theorems, Cambridge University Press, London, UK, 1980. 